\title{
Comparative analysis of Long-Haul System based on SSB modulation Utilising Dual Parallel Mach- Zehnder Modulators
}

\author{
Fadil Paloi, and *Shyqyri Haxha \\ Department of Electronic Engineering, Royal Holloway, University of London \\ Egham, Surrey, TW20 0EX, UK \\ Email:*Shyqyri.Haxha@rhul.ac.uk
}

\begin{abstract}
In this paper, we have proposed a long-haul optical transmission system, based on a single sideband (SSB) modulation scheme. Analytical and simulation models have been developed, optimised and demonstrated for the proposed SSB system configurations. The SSB modulation scheme was proposed to overcome dispersion in the fibre. We have shown that the related link losses can be minimized by increasing the quality of the optical signal at the modulation. We have optimised the radio over fibre (RoF) configuration scheme based on Dual Parallel Dual Drive Mach Zehnder Modulator (DP-DDMZM), thereby increasing transmission length of the fibre. With the proposed SSB, by suppressing some of the harmonics and cancelling one of the sidebands, we have halved the RF power fading and interference. The developed analytical (theoretical/mathematical) model agrees very well with the simulation results using two (both) different commercial simulation tools. The optical signal is boosted while minimizing the number of repeaters. We report a SSB configuration, compensation and amplification with individual spans of $150 \mathrm{~km}$, by extending the length of the link up to $3250 \mathrm{~km}$. The proposed system configuration exhibits high performance with less complexity and lower cost.
\end{abstract}

Keywords: Fibre optics communications, Radio Over Fibre, Single Sideband Modulation, Single Mode Fibre.

\section{Introduction.}

With rapid growth in demand of the high capacity wired and wireless signals, optical fibre for the long-haul transmission has provided a huge bandwidth and has become crucial for the signal transmission (Hayashi et al. 2017; Mizuno et al. 2017). The main tasks emerging for designers of long-haul systems are losses at the modulation and nonlinear impairments in the Single Mode Fibre (SMF) (Wei et al.2017; Haxha et al. 2008 and Hirano et al. 2009). To some degree, this loss can be recovered by increasing the quality of the optical signal at the input, i.e. correct modulation. The transmission of the RoF system, over the fibre optic link, is evidently controlled by the carrier (Niknamfar and Shadaram 2013). Nonlinear distortion can occur due to the power fading and interference of the two beats created between each sideband and the optical carrier of the double-sideband signal. Thus, to minimise the linked RF power fading effect and interference, harmonics must be suppressed and one of the sidebands must be cancelled (Phillips and Darcie 1997; Chen and Yao 2016).

In this study, we have investigated several RF over fibre configuration structures, and we have proposed a new configuration structure, where key performance parameters such as the RF power requirement and maximum transmission distance have been optimised (Miziya et al. 2013; Gupta et al. 2012; Paloi et al. 2017).

Our aim is to explore SSB configurations within existing optical fibre links that would boost the signal, increasing the data transmission distance, reducing the system complexity and reducing the number of amplifiers. We have proposed modulation which incorporates DP-DDMZM and provides the advantage of overcoming penalties due to chromatic dispersion. Prior to generating simulation results using VPI and Optisystem simulation software, we have benchmarked our simulations with experimental models published (Jiang et al. 2015; Devgan et al. 1012), based on the linearization and harmonic distortion of the photonic links. We have compared this work with dispersion compensation schemes (Paloi et al. 2017; Xu and Ou 2015).

Let us consider the child Mach-Zehnder Modulator (MZM). If we configure the first child modulator for the pushpull operation, at the Quadrature Transmission Point (QTP), this will result in higher gain and minimised even order distortion. If the second child MZM is also configured for the push-pull operation, at the minimum transmission point (mTP), this results in low gain and low spur product, which means high SFDR. We have used three compensation structures; pre, in-line symmetrical, and post-compensated scheme. These can compensate any nonlinearities left from the modulation, can compensate the fibre impairments caused by the length of the fibre such as amplified spontaneous emission (ASE), and can compensate left fibre losses by preventing nonlinearities going through the link to the receiver. In Long-haul transmission, the minimum use of repeaters is desirable. The SSB modulation scheme has been optimised to minimise the number of repeaters per unit distance (Patel et al. 2014). The proposed SSB configuration can be implemented within existing fibre links. Compensation and amplification have been used after $150(\mathrm{~km})$ for each span, whilst maintain a stable signal. By using the SSB scheme and dispersion compensation, we have managed to boost the optical link length up to 3250 ( $(\mathrm{km})$, and lower complexity. The proposed SSB configuration compare to previous implementations (Miziya et al. 2013; Gupa et al. 2012 and Paloi et al. 2017), shows more robustness against the losses, and we have effectively extended the reach of the signal transmission using existing hardware. 


\section{Methodology}

Our chosen optical modulation format is on-off-keying (OOK) RZ (Chen et al. 2016 and Miziya et al. 2013). We have investigated the system link by upgrading a RoF configuration scheme with a SSB scheme. The chromatic dispersion can successfully be compensated by using dispersion compensation fibre (DCF) (Breuer et al.1997). In a modulator, adjustment of the bias voltage plays a vital role for the chosen operating point. The transmission operating point significantly affects the system's performance, particularly impacting the harmonic distortion (Svarny et al. 2013). The DC voltage applied to a modulator is called bias voltage. The bias works by changing the refractive index and will lead to an optical phase shift between the two optical paths, causing different amounts of optical interference at the Y coupler combiner of the MZM.

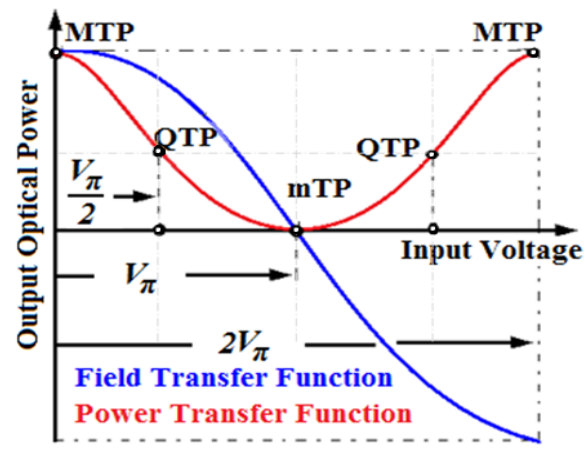

Fig. 1. MZM transfer chart at various transmission point

To have maximum intensity at the output of MZM, we must generate a constructive recombination which is a zero phase-shift between the two paths. By gradually increasing the phase difference between these two paths at the output of the MZM, the amount of destructive interference will increase (Li et al. 2013).

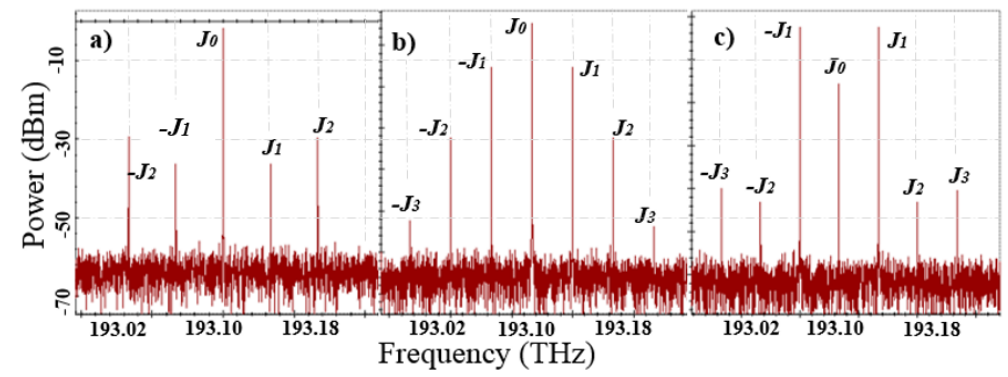

Fig. 2. Output optical spectrum of MZM at various transmission point; a) Maximum Transmission Point (MTP). b) Quadrature Transmission Point (QTP) c) Null or minimum transmission point (mTP).

The MZM transfer functions or operating points at the different bias voltages for the DP-DDMZM are identified, as illustrated in Fig.1 and Fig.2.

The Maximum Transmission Point (MTP), with $\mathrm{V}_{\text {bias }}=0$, or $2 \pi$, as shown in Fig. 1, results in high second order harmonic and therefore poor Spurious-Free Dynamic Range (SFDR). Most of the lights are thrown away, as shown in Fig. 2a.

The Quadrature Transmission Point (QTP), with $\mathrm{V}_{\text {bias }}=\pi / 2$, results in high gain and minimized even order distortion for small signals, as illustrated in Fig. $2 b$.

Null or minimum transmission point $(\mathrm{mTP})$, with $\mathrm{V}_{\text {bias }}=\mathrm{V} \pi$, results in low gain, low noise figure (low spur product) and high SFDR, as illustrated in Fig. 2c.

\section{Performance criteria}

Bit error ratio (BER) is the key performance parameter evaluating the performance of the data link. BER is estimated through numerical simulation expressed in terms of the Quality factor, corresponding with eye diagrams. The $\mathrm{Q}$ factor is measure of optical signal to noise ratio for binary signal. It is ratio between the difference of the optical signal intensity of the higher " 1 " and lower level " 0 " in an eye diagram, and the sum of RMS noise on each edge of the eye. (Freude et al. 2012).

$$
Q=\frac{\mu_{1}-\mu_{0}}{\sigma_{1}+\sigma_{0}} ; \quad B E R=\frac{1}{2} \operatorname{erfc}\left(\frac{Q}{\sqrt{2}}\right) \approx \frac{\exp (-Q / 2)}{Q \sqrt{2 \pi}}
$$


Another metric of link performance is modulation index. Modulation index is a ratio between applied signal amplitude voltage and switching voltage in a specified modulated signal. It is a key parameter for any modulation, showing the level of modulated signal (Hu et al. 2006).

$$
m=\pi \frac{V_{R M S}}{V_{\pi}}=\pi \cdot M_{d}
$$

where, $m$ is modulation index and $M d$ is modulation depth. Maximum modulation depth is $\pi$ and converted in to the modulation index is " 1 ", which is $100 \%$ modulation (Yu et al. 2013).

\section{Proposed ROF Optical Configuration System}

We have proposed a RoF system configuration for a fibre length of $120(\mathrm{~km})$ to $3250(\mathrm{~km})$ with a bit rate of 10 (Gbit/s) to 100 (Gbit/s). The system configuration illustrated in Fig. 3 covers transmitter, fibre optic link and receiver. The main components in the transmitter are; continuous wave $(\mathrm{CW})$ laser of $1550 \mathrm{~nm}$, with line width of 0.1(MHz), linked with RF signal on the DP-DDMZMs, which covers various compensation schemes developed by (Miziya et al. 2013; Gupta et al. 2012; Paloi et al. 2017) to overcome the dispersion in the fibre and receiver. We will assume a cut off frequency of 0.75 (bitrate). In the RoF system, the fibre dispersion induced power fading can be resolved by using the OSSB (Niknamfar and Shadaram 2013; Cui et al. 20012).

Prior to generating simulation results using VPI and Optisystem simulation software, we benchmarked our simulations with experimental results published (Devgan et al 2012; Xu et al. 2015), based on the linearization and harmonic distortion of the photonic link and other published work for dispersion compensation (Paloi et al. 2017; $\mathrm{Xu}$ et al. 2015). When we compare our results with other published based modulation schemes, the maximum achieved transmission length goes up to $2400(\mathrm{~km})$, as illustrated in Fig. 9a. The reduced range in a conventional system is caused by distortion at the modulation. The RoF increase in range was affected by the SSB modulation. The fibre dispersion induced power fading has been minimised by creating an SSB, which is formed with low distortion products, obtaining much higher efficiency and increasing transmission distance of up to 3250(km) (Niknamfar et al. 2013; Cui et al. 2012), as illustrated in Fig. 9b. The link parameters used by both configurations have certain similarities such as; Standard Single Mode Fibre (SSMF), with attenuation of 0.2 $(\mathrm{dB} / \mathrm{km})$ and dispersion of 16 [ps/(nm. km)]. The DCF is used as a dispersion compensator, per the link length, with attenuation of $0.5(\mathrm{~dB} / \mathrm{km})$ and dispersion of -80 [ps/(nm.km)] (Paloi et al. 2017; Eappen et al 2014). Each fibre span is amplified per the transmission length with Erbium-Doped Fibre Amplifier (EDFA) and 6(dB) noise figure. We have based our analysis on the parameters of the standard Corning SMF-28 for the mode area of 75 $\left(\mu \mathrm{m}^{2}\right)$.

\subsection{Single Sideband (SSB) modulation scheme}

The use of optical single sideband (OSSB) modulation with an optimum sideband to spur ratio (Dynamic Range) will improve spectral efficiency by putting more of the optical power into useful light. The OSSB modulation can be achieved by removing one of the sidebands with precise shifting of the DP-DDMZM.

The proposed SSB modulation scheme illustrated in Fig. 3 consists of two standard DD-MZM, assumed to be identical and are driven by the same electrical signals.

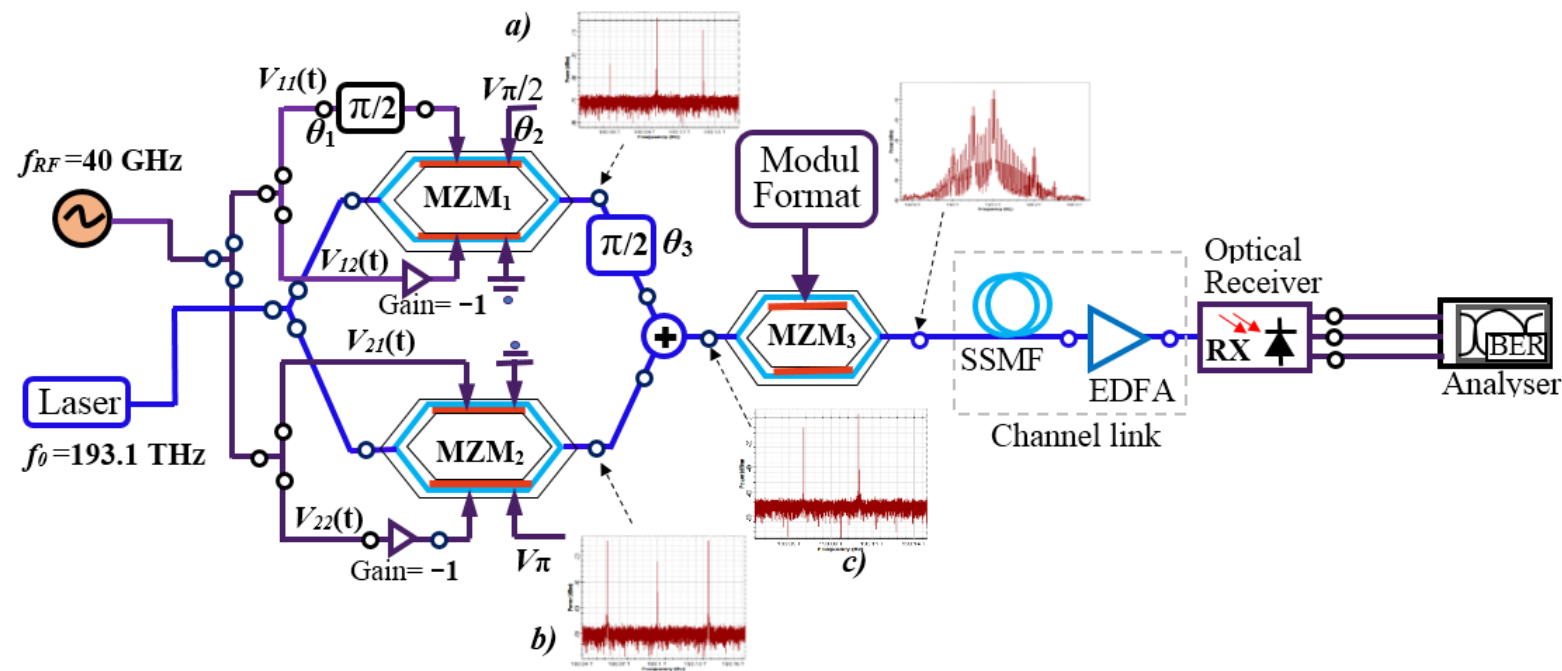

Fig. 3. Proposed SSB configuration, using DP-DDMZM configured with first child MZM at the QTP, and second child MZM at the mTP, both at the push-pull operating mode, SSMF, EDFA, Optical receiver (RX), Bit error ratio analyzer (BER). 
A CW Laser is used as a light source, separated in to two branches by a 3(dB) optical splitter, directed in to both upper and lower DD-MZMs. The input RF message is a sinusoidal signal divided into two paths for each modulator, with RF upper $90^{\circ}$ shifted. Both modulators are configured at the push-pull configuration mode and optically shifted from each other by $90^{\circ}$, as illustrated inFig.3.

With proper electrical phase delay $\theta_{1}(\mathrm{RF}), \theta_{2}$ (bias) combination at the input of both child MZMs, and other optical phase delays, we have suppressed some of the harmonics and achieved an SSB Modulation at the output of parent MZM. Both parallel modulators have a DC voltage $V_{\pi}$ of $4(V)$, voltage required to go from destructive to constructive optical interference at the combining coupler and causes phase shift of the light by one half of the optical wavelength comparative to the other. This phase shift also corresponds to the on/off voltage of the MZM. The MZM has an extinction-ratio of $30(\mathrm{~dB})$ and insertion losses of $8(\mathrm{~dB})$. The electric field of the light from a Continuous Wave Laser (CWL) at frequency of 193.1 (THz) is represented at the complex form (Xue et al 2014):

$$
E_{0}(t)=\sqrt{2 k P_{0}} e^{j \omega_{0} t}=E_{0} e^{j \omega_{0} t}
$$

where $P_{0}$ is the average laser power, constant $k$ related to the field and average power, $E_{0}$ is electric field amplitude with an angular frequency $\omega_{0}$. Fig. 3 shows the main scheme of the link, where various MZM techniques can be employed within its non-linear transmission curve, as shown in the Fig 1. It is assumed that both MZMs are identical, with same driving RF signal voltages, and the same push-pull configuration at different operating points. The main scheme is divided in to two modulation schemes where both have been simulated and investigated separately, as illustrated in Fig. 4, and Fig. 6.

$\mathrm{MZM}_{1}$ is operating at the QTP, with biased DC voltage of $V_{\pi} / 2=2(V)$ for first branch and second one grounded, as illustrated in Fig.4. Optical output of the $\mathrm{MZM}_{1}$ is delayed for $\theta_{3}=\pi / 2$ or by shifting both MZMs for $\pm \pi / 4$.

DD-MZM 1 is a photonic mixer, where instant value of the modulating signal is applied to the upper and lower electrodes, as illustrated in Fig. 4.

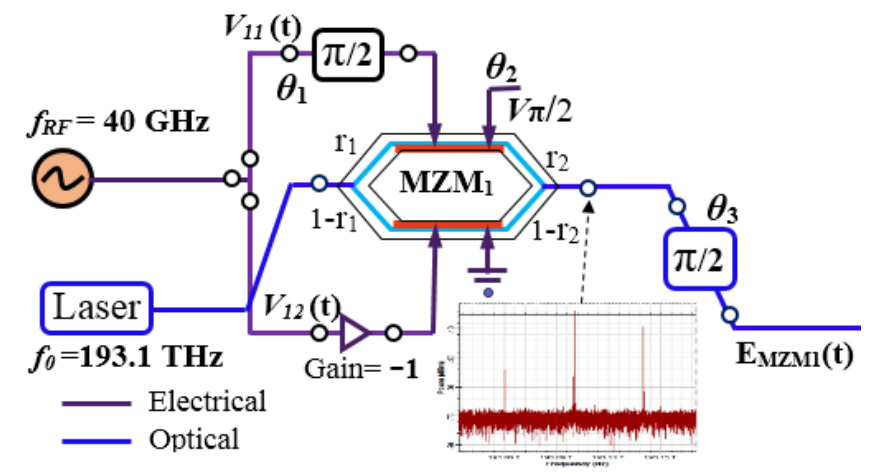

Fig. 4. Schematic configuration of DD-MZM1, generating Vestigial Sideband signal.

The RF modulating electrical voltages $V_{11}(t)$ and $V_{12}(t)$ can be expressed as two sinusoidal signals with the power of $14(\mathrm{dBm})$. The bias voltages $V_{\text {bias1 }}$ and $V_{\text {bias2 }}$ are voltages applied to both arms of the DD-MZM 1 , can be applied differentially via the voltages $V_{\mathrm{dc} 1}$ and $V_{\mathrm{dc} 2}$ compared to the single drive, as illustrated in Fig. 4 , and can be expressed as (Xue et al. 2014):

$$
\begin{aligned}
& V_{11}(t)=V_{11} \cos \left(\omega_{R F} t+\theta_{1}\right)+V_{\pi} / 2=-V_{11} \sin \omega_{R F} t+V_{\pi} / 2 \\
& V_{12}(t)=V_{12} \cos \left(\omega_{R F} t+\pi\right)=-V_{12} \cos \omega_{R F} t+0
\end{aligned}
$$

Where $V_{11}$ and $V_{12}$ are the signal amplitudes of the upper and lower arm of the $\mathrm{MZM}_{1}, \omega_{R F}$ is the angular frequency of the RF signal, and $\theta_{1}=\pi / 2$ is the phase difference between signals of the upper and lower arms, as shown in Fig. 4. The optical phase difference existing in the arms of the $\mathrm{MZM}_{1}$ through the linear electro optic effect based on Ref. (Li et al. 2013) can be expresses as:

$$
\begin{aligned}
& \Phi_{11}(t)=\pi \frac{V_{11}(t)}{V_{\pi}}=-\pi \frac{V_{11}}{V_{\pi}} \sin \omega_{R F} t+\pi \frac{V_{\pi d c}}{2 V_{\pi}} \\
& \Phi_{12}(t)=\pi \frac{V_{12}(t)}{V_{\pi}}=-\pi \frac{V_{12}}{V_{\pi}} \cos \omega_{R F} t
\end{aligned}
$$




$$
\Phi_{\text {Bias }}(t)=\pi \frac{V_{\text {Bias }}}{V_{\pi}}=\theta_{2}=\frac{\pi}{2}
$$

$\theta_{2}-$ is the phase difference caused from the biased voltage applied to the $\mathrm{MZM}_{1}$. From all simulations that can be seen, bias voltage affects the Optical Signal to Sideband Ratio (OSSR). For the ideal bias voltage, we will get maximum OSSR, but when bias voltage deviates from the desired value, OSSR will decline noticeably.

The optical power delivered by the laser reaches the input junction of the DD-MZM 1 with the $\mathrm{LiNbO}_{3}$ substrate, per an equivalent splitting ratio (Lin et al. 2008; Ferreira et al. 2012).

$$
E_{M Z M 1}(t)=E_{0} e^{j \omega_{0} t} \cdot\left\{\begin{array}{l}
\sqrt{r_{1} r_{2}} e^{j \Phi_{1}(t)} \\
+\sqrt{\left(1-r_{1}\right)\left(1-r_{2}\right)} e^{\left.j \Phi_{12} t t\right)}
\end{array}\right\} \cdot e^{j \pi \frac{V_{\pi}}{2 V_{\pi}}}
$$

If the power transmission coefficients are $r_{l}=r_{2}$, we have 50/50 ideal power splitting (Dattoli et al. 1998). If this parameter deviates from the ideal value, the optical signal to sideband ration will be affected. The 50/50 splitting ratio at the DD-MZM is particularly difficult to be achieved but in most cases, it is assumed as a balanced splitting ratio.

Based on the chirp modelling for the DD-MZM, when the modulator operates at the QTP, bias voltage places the modulator at the midpoint of the optical non-linear transmission curve, as shown in Fig. 1. This means intensity is at half of its peak value. If chirp is given in terms of driving voltage:

$$
\alpha=\frac{V_{1}+V_{2}}{V_{1}-V_{2}}
$$

To achieve zero chirp $(\alpha=0)$ from the DD-MZM, voltages applied to the upper and lower arm should be equivalent $V_{l}=-V_{2}$, achieving pure intensity modulation with no phase modulation, or $V l=V 2$ we will achieve pure phase modulation $\alpha=\infty$, (Kaur et al. 20011).

The mathematical calculation of the output optical field for the $\mathrm{MZM}_{1}$ can be expressed as:

$$
\begin{aligned}
& E_{M Z M 1}(t)=\frac{E_{0}}{2} e^{j \omega_{0} t} \cdot \\
& \left\{e^{-j \pi \frac{V_{R M S}}{V_{\pi}} \sin \omega_{R F} t} \cdot e^{j \pi \frac{V_{\pi d c}}{2 V_{\pi}}}+e^{-j \pi \frac{V_{R M S}}{V_{\pi}} \cos \omega_{R F} t}\right\} e^{j \frac{\pi}{2}}
\end{aligned}
$$

Equation (9) shows theoretical analysis for the upper and lower paths of $\mathrm{MZM}_{1}$, where the fields for the carrier and sidebands, controlled by bias relationship, will determine SSB generation.

$$
m=\pi \frac{V_{R M S}}{V_{\pi}}=\pi \frac{V_{p}}{\sqrt{2} V_{\pi}}
$$

Where " $m$ " is modulation index, which is a ratio between applied signal amplitude voltage $V_{p}$ or $V_{R M S}$, to the switching voltage " $V_{\pi}$ ". Where $V_{p}$ is peak input RF voltage and $V_{R M S}$ is root mean square (RMS) voltage, applied after the splitter, account for the loss in the splitting, from peak value of $3 \mathrm{dBm}$.

The modulated signal of DDMZM 1 can be expressed as (Thomas et al. 2016):

$$
E_{M Z M 1}(t)=\frac{E_{0}}{2}\left\{\begin{array}{l}
\cos \omega_{0} t \cdot \sin \left(m \cos \omega_{R F} t\right) \\
-\cos \omega_{0} t \cdot \cos \left(m \sin \omega_{R F} t\right) \\
-\sin \omega_{0} t \cdot \sin \left(m \cos \omega_{R F} t\right) \\
-\sin \omega_{0} t \cdot \cos \left(m \sin \omega_{R F} t\right)
\end{array}\right\}
$$

Equation (11) can be solved by using the Bessel function identities (Dattoli et al. 1998), analysing the sidebands to produce a solution. By using the Jacobi-Anger identity, the output field of the first modulator is achieved: 


$$
\mathrm{E}_{\mathrm{MZM} 1}(t)=\frac{\mathrm{E}_{0}}{\sqrt{2}} \cdot\left\{\begin{array}{l}
-J_{0}(m) \cos \omega_{0} t \\
+J_{1}(m) \cdot\left[\sin \left(\omega_{0} t+\omega_{R F} t\right)+\sin \left(\omega_{0} t-\omega_{R F} t\right)\right] \\
-J_{2}(m) \cdot\left[\sin \left(\omega_{0} t+2 \omega_{R F} t\right)+\sin \left(\omega_{0} t-2 \omega_{R F} t\right)\right] \\
-J_{3}(m) \cdot \cos \left[\left(\omega_{0} t+3 \omega_{R F} t\right)+\cos \left(\omega_{0} t-3 \omega_{R F} t\right)\right]
\end{array}\right\}
$$

As can be seen from equation (12), $J n(m)$ functions are known as Bessel function of first kind, which in our case are decreasing sine and cosine functions.

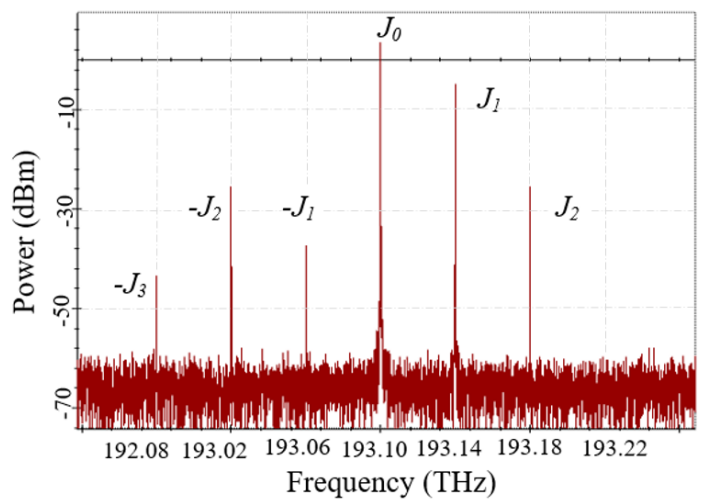

Fig. 5. Optical VSSB spectrum performed at the output of first child modulator $\left(\mathrm{MZM}_{1}\right)$, created from phase shift of $\theta 1=\pi / 2$, push-pull configuration and, $V \pi / 2 \mathrm{dc}$ biasing of one of electrodes

The zero order Bessel function $J_{0}(m)$ determines the amplitude of the carrier wave, and higher orders such as $J_{n}(m)$ determine the amplitude of the $n^{\text {th }}$ pair of the sidebands. In the first scheme, demonstrated in Fig. 5, the proportion of AM compared to PM is optimised for only a specific frequency, which produces Optical Vestigial Single Sideband (OVSS). During our simulation, we note that the second and third order harmonics cannot be cancelled equally at the same time. In this case, we have set the bias condition at QTP to suppress the third order harmonic. This will cause the second order intercept to decrease noticeably, securing OVSSB transmission through the configuration link shown in Fig. 4, where obtained results are illustrated in Fig. 5.

In addition, by setting the bias condition at mTP, for the second $\mathrm{DDMZM}_{2}$ (configuration link, Fig. 6), with DC voltage of $V_{\pi}=4(V)$, we have achieved a carrier suppressed signal spectrum with the second order harmonics suppressed, leading the third order intercept to decrease noticeably, as shown in the Fig. 7 (Lin et al. 2008).

The phase of the upper and lower arms of the $\mathrm{MZM}_{2}$ are shifted because of the applied $V_{\pi} / 2 \mathrm{DC}$, as illustrated in Fig. 6 (Ferreira et al. 2012).

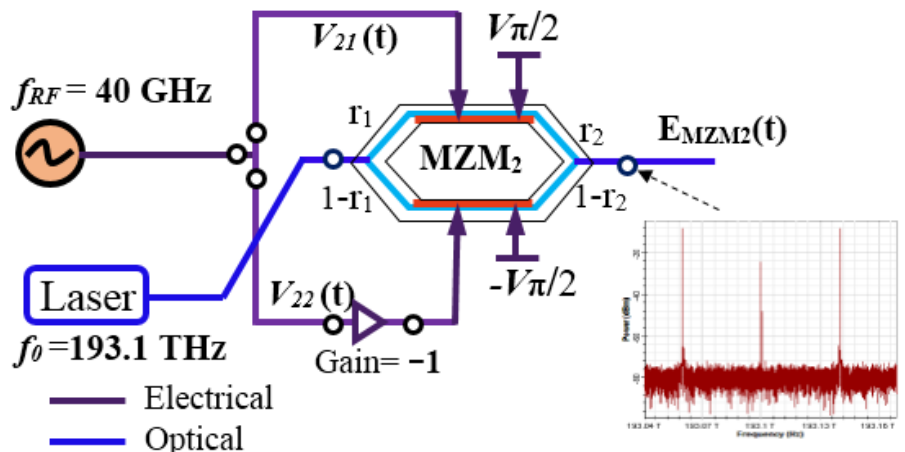

Fig. 6. Schematic configuration of DD-MZM 2 , performing Carrier Suppressed Sideband (CSSB) signal, at the push-pull configuration and, $\pm V_{\pi} / 2$ DC biasing of each electrodes.

$$
\begin{aligned}
& V_{21}(t)=V_{21} \cos \omega_{R F} t+V_{\pi} / 2 \\
& V_{22}(t)=-V_{22} \cos \omega_{R F} t-V_{\pi} / 2
\end{aligned}
$$




$$
\begin{aligned}
& \Phi_{21}(t)=\pi \frac{V_{21}(t)}{V_{\pi}}=\pi \frac{V_{21}}{V_{\pi}} \cos \omega_{R F} t+\pi \frac{V_{\pi d c}}{2 V_{\pi}} \\
& \Phi_{22}(t)=\pi \frac{V_{22}(t)}{V_{\pi}}=-\pi \frac{V_{22}}{V_{\pi}} \cos \omega_{R F} t-\pi \frac{V_{\pi d c}}{2 V_{\pi}}
\end{aligned}
$$

Optical power delivered by the laser will reach the input junction of the DD-MZM $\mathrm{M}_{2}$ with the transmission coefficient of $r_{1}=r_{2}$, as refer to Eq. (7), (Rogers et al. 2010).

$$
\begin{gathered}
E_{M Z M 2}(t)=\frac{E_{0}}{2} e^{j \omega_{0} t}\left\{e^{j \Phi_{21}(t)}+e^{j \Phi_{22}(t)}\right\} \\
E_{M Z M 2}(t)=\frac{E_{0}}{2} e^{j \omega_{0} t} \cdot \\
\left\{e^{j\left[\pi \frac{V_{21}}{V_{\pi}} \cos \omega_{R F} t+\pi \frac{V_{\pi d c}}{2 V_{\pi}}\right]}+e^{-j\left[\pi \frac{V_{22}}{V_{\pi}} \cos \omega_{R F} t+\pi \frac{V_{\pi d c}}{2 V_{\pi}}\right]}\right\}
\end{gathered}
$$

For push-pull configuration; $V_{21}(t)=-V_{22}(t)$.

$$
\begin{gathered}
E_{M Z M 2}(t)=E_{0} e^{j \omega_{0} t} \cdot \\
\left\{\begin{array}{c}
\cos \left(\pi \frac{V_{d c 21}-V_{d c 22}}{2 V_{\pi}}+\pi \frac{V_{21}(t)-V_{22}(t)}{2 V_{\pi}}\right) \\
\left.e^{j\left[\pi \frac{V_{d c 21}+V_{d c 22}}{2 V_{\pi}}+\pi \frac{V_{21}(t)+V_{22}(t)}{2 V_{\pi}}\right]}\right\}
\end{array}\right\} \\
E_{M Z M 2}(t)=E_{0} e^{j \omega_{0} t} \cdot\left\{\cos \left(\frac{\pi}{2}+\pi \frac{V_{R M S}}{V_{\pi}} \cos \omega_{R F} t\right)\right\} \\
m=\pi \frac{V_{R M S}}{V_{\pi}}=\pi \frac{V_{p}}{\sqrt{2} V_{\pi}} \\
E_{M Z M 2}(t)=E_{0} \cdot\left\{\begin{array}{l}
-\cos \omega_{0} t \cdot \sin \left(m \cos \omega_{R F} t\right) \\
-j \sin \omega_{0} t \cdot \sin \left(m \cos \omega_{R F} t\right)
\end{array}\right\}
\end{gathered}
$$

Equation (19) can be solved by using the Bessel function identities where, after Jacoby-Anger expansion, the output optic field for the bottom $\mathrm{MZM}_{2}$ can be written as follows:

$$
E_{M Z M 2}(t)=E_{0}\left\{\begin{array}{l}
-J_{1}(m) \cdot\left[\cos \left(\omega_{0} t+\omega_{R F} t\right)+\cos \left(\omega_{0} t-\omega_{R F} t\right)\right]+ \\
J_{3}(m) \cdot\left[\cos \left(\omega_{0} t+3 \omega_{R F} t\right)+\cos \left(\omega_{0} t-3 \omega_{R F} t\right)\right]
\end{array}\right\}
$$

Referring to Eq. (20), the optical signal consists of the first and third sidebands, with suppressed carrier, where, $J_{l}(\mathrm{~m})$ and $J_{3}(\mathrm{~m})$ are represent the first and third order sideband at the Bessel function of first kind, (Lin et al. 2008).

Optical carrier suppressed signal spectrum is performed at the second chid modulator Fig. 6, and illustrated in Fig. 7, with in phase mode $-J_{l}(m)$ and $J_{3}(m)$ propagating through the " $Y$ " junction as output signal, and rest as the out of phase, $J_{l}(m)$ and $-J_{3}(m)$, radiating from the waveguide at the " $Y$ " junction (Shimotsu et al. 2001). The second and third order harmonics are showing, because in practice we cannot rely on complete harmonic cancellation. This is due to some optical imbalance and high input power within the MZM, for example some phase-modulated light gets through, known as Chirp. Extinction Ratio (ER) is also unlikely to be the same for both child modulators. 


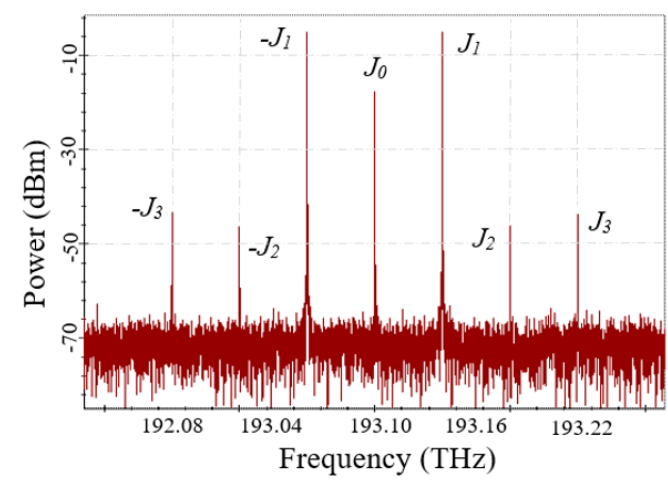

Fig. 7: Optical CS-SB spectrum performed at the second child modulator $\left(\mathrm{MZM}_{2}\right)$, at the push-pull configuration and, $\pm V \pi / 2 \mathrm{dc}$ biasing of each electrode.

Next, we combine these two DD-MZMs.

$$
\begin{gathered}
E_{\mathrm{DP}-\mathrm{DDMZM}}(t)=E_{M Z M 1}(t)+E_{M Z M 2}(t) \\
E_{D P-D D M Z M}(t)=\left\{\begin{array}{l}
-\frac{\sqrt{2}}{2} J_{0}(m) \cos \omega_{0} t-\sqrt{2} J_{2}(m) \sin \omega_{0} t \cdot \cos 2 \omega_{R F} t \\
-\sqrt{2} J_{1}(m) \cos s \omega_{0} t \cdot \cos \omega_{R F} t+ \\
\sqrt{2} J_{3}(m) \sin \omega_{0} t \cdot \cos 3 \omega_{R F} t
\end{array}\right\}
\end{gathered}
$$

With parallel combination of two DD-MZM and with appropriate bias control for each modulator, based on Eq. (21), we have managed to completely remove the third order $\left(-J_{3}\right)$ sideband and suppress the other third order sideband $\left(J_{3}\right)$, while generating the OSSB $\left(-J_{l}\right)$ needed for the long-haul transmission, as illustrated in Fig. 8a, $(\mathrm{Xu}$ et al. 2015). The SSB signal generated after the parent modulator is connected to the third modulator, where the output optical signal will modulate coded messages, as illustrated in Fig.8b.
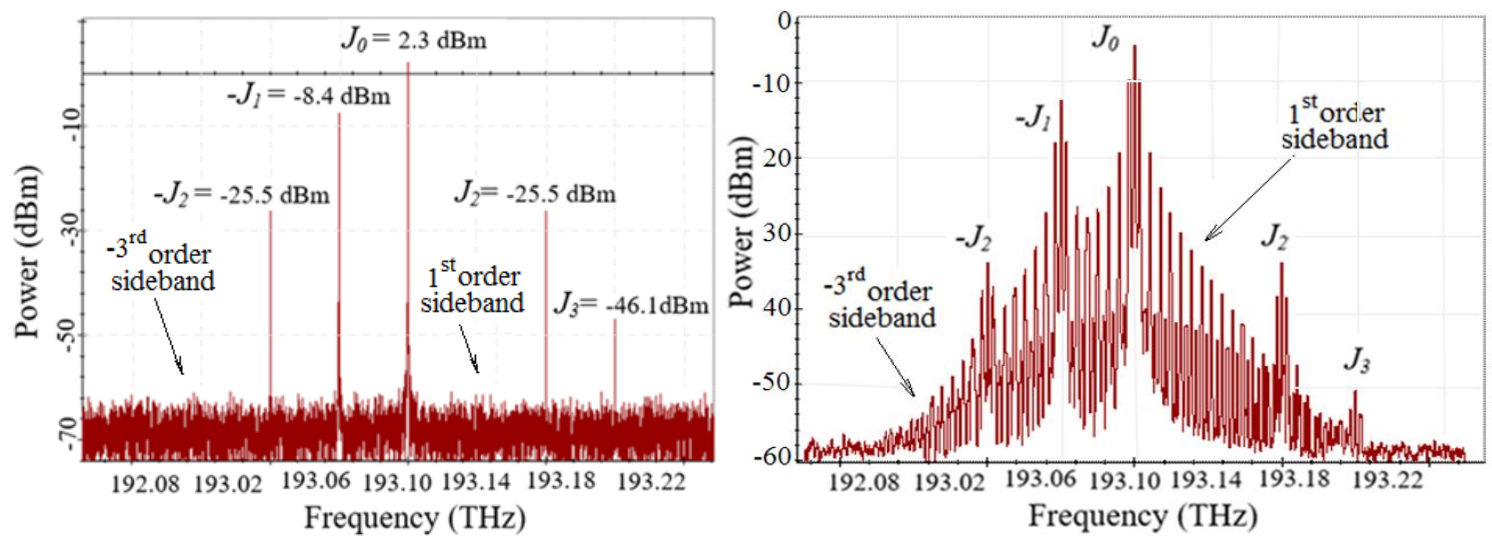

Fig. 8: Optical SSB spectrum performed at the: a) Parent MZM, and b) After MZM3, presenting harmonic spectra consistent with a modulation-depth ' $m$ '.

To find out whether the simulation model and mathematical analysis matches, we calculated the modulation index at different input values.

At the RF, the peak power of $P_{(w)}=10(\mathrm{~mW})$, for $V_{\pi}=4(\mathrm{~V})$, into modulators input resistance of $R=50(\Omega)$, we will calculate the modulation index.

$$
P_{w}=\frac{\left(\frac{V_{p}}{\sqrt{2}}\right)^{2}}{R} \text { where: }
$$




$$
\begin{gathered}
V_{R M S}=\frac{V_{P}}{\sqrt{2}}=\frac{\sqrt{P_{w} \cdot 2 R}}{\sqrt{2}}=\frac{1}{\sqrt{2}}=0.7071[V] \\
m=\pi \frac{V_{P}}{\sqrt{2} V_{\pi}}=\pi \frac{V_{R M S}}{V_{\pi}}=0.555
\end{gathered}
$$

The actual harmonic spectra illustrated in Fig.8a are consistent with a modulation-depth ' $m$ ' factor of 0.555 for the $V_{\text {peak }}=1(V)$, or $V_{R M S}=0.707(V)$ and $V_{\pi}=4(V)$, where calculated $\mathrm{Jn}$ (Bessel) coefficients are:

$$
\begin{gathered}
J_{0}(m)=20 \log _{10}(0.924)+3=2.3 \mathrm{dBm} \\
J_{1}(m)=20 \log _{10}(0.267)+3=-8.4 \mathrm{dBm} \\
J_{2}(m)=20 \log _{10}(0.0375)+3=-25.5 \mathrm{dBm} \\
J_{3}(m)=20 \log _{10}(0.0035)+3=-46.1 \mathrm{dBm}
\end{gathered}
$$

The agreement between theory and simulated data is satisfied once $3 \mathrm{~dB}$ was added to account for the loss in the modulation, as seen in chart Fig. 8a. Thus, the result is consistent with our VPI simulations. Furthermore, to analyse the transmission performance of the generated OSSB signal, we have performed a comparison between the standard modulation scheme carried out by (Paloi et al. 2017), and the proposed OSSB modulation in a RoF System link at various distance at the SSMF, using a combination of the three compensation channel link schemes used by (Paloi et al. 2017).

\section{Performance Analysis}

In this section, we illustrate the performance of the DD-MZM. Based on the Ref. (Gupa et al. 2012; Paloi et al. 2017 and Eappen et al. 2014), we are focused on the system configuration with the main aim being to enhance the system performance at the modulation by removing as much power fading and interference as possible, before entering the fibre optic link. We must comprehend that the distortion caused due to the CD is obvious if the link is not accurately compensated. The CD will cause each spectral component to phase shift with respect to each other as they propagate down the fibre. At the fibre optic channel link, we have used a combination of three dispersion compensation maps used by; (Paloi et al. 2017) to overcome the dispersion in the fibre.

The Q Factor of the proposed structure is plotted in function of the fibre length for 10 to $100 \mathrm{Gbit} / \mathrm{s}$ bitrates, for both configurations. The BER is specified as $10^{-9}$, corresponding the value of Q-Factor of 6 .

Comparing both configurations, and from the achieved analytical and graphical results, the proposed SSB configuration shows more robustness against the losses, as illustrated in Fig. 9a, compare to proposed configuration Fig. 9b, (Miziya et al 2013; Gupa et al. 2012; Paloi et al. 2017).
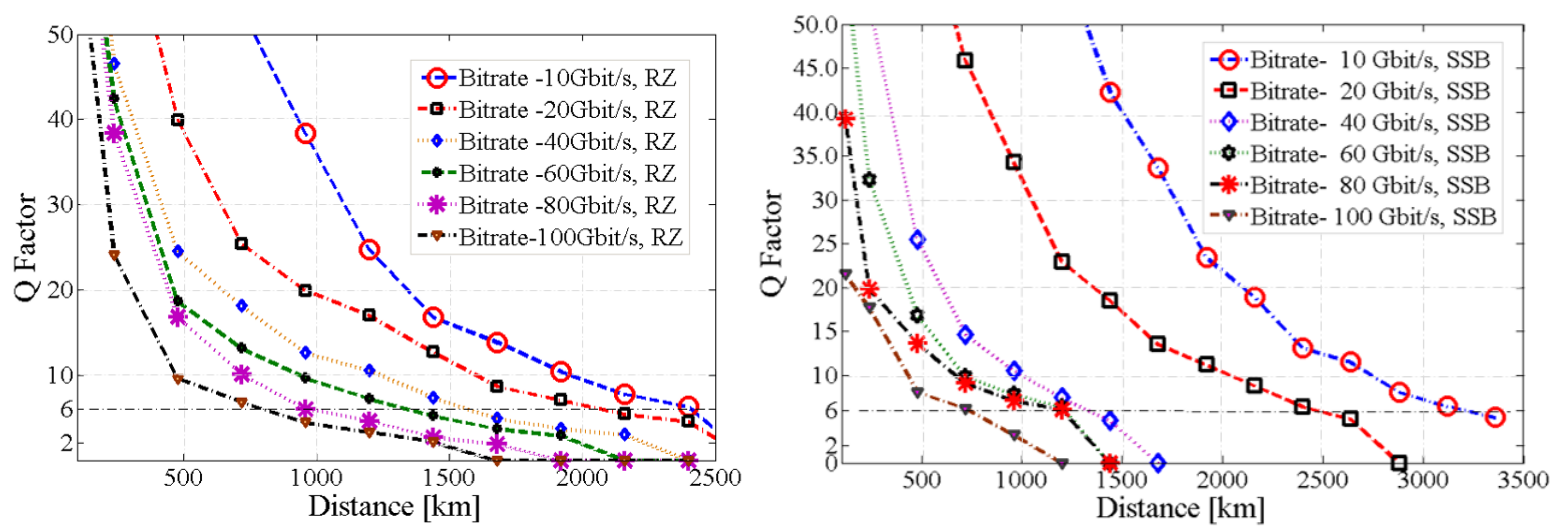

Fig. 9. Measured Q-Factor versus fibre propagation distance, after various bitrates, a) Using conventional RZ modulation scheme b) using proposed SSB modulation scheme.

Our analysis of the proposed theoretical and simulation model demonstrates that with proper shifting, by correctly setting the bias point at the DP-DDMZM, and by applying appropriate DCF at the channel link, we have halved the related RF power fading and interference, and minimised losses at the system link respectively.

This has resulted in the suppression of third order harmonics $J_{3}$, completely cancelling the other third order $-J_{3}$ sideband, while generating the OSSB $-J_{l}$ needed for the long-haul transmission as shown in Fig. 8(a and b).

By applying this settings to the proposed SSB configuration, we have managed to extend the transmission length from $2400(\mathrm{~km})$ done by (Paloi et al. 2017) Fig. 9a to $3250(\mathrm{~km})$, Fig. 9b. 
Every time we have amplified signal, nonlinear distortion when traveling through the link have been boosted. Therefore, at the beginning of the link a pre-compensation scheme is employed, having the ability to compensate any nonlinear distortion left from the modulation (Paloi et al. 2017). The EDFA adds to the noise power spectral density (PSD) because of additive noise factor such as the ASE, (Kaur et al. 2011). Each spectral component will experience different delay because of fibre dispersion and the total relative phase shifting will depend on the length of the fibre. With a proper constructed in-line symmetrical compensation scheme, we have minimized the fibre impairments such as CD, caused from the long-length of the fibre (Patel et al. 2014). As is evident from Fig. 9a and Fig. 9b, transmission for a data rate of 10 to 100 (Gbit/s) is assessed. The proposed SSB modulation scheme, as compared to conventional configuration used by (Miziya et al 2013; Gupa et al. 2012; Paloi et al. 2017), appears to be more robust against the losses. This gives us better results, where for the bitrate of $10(\mathrm{Gbit} / \mathrm{s})$ and Q-Factor $=6$, potentially reaching the transmission distance of $3250(\mathrm{~km})$.

Likewise, for bitrates of $20(\mathrm{Gbit} / \mathrm{s})$ reaches transmission distance of $2550(\mathrm{~km})$ and when bitrate is increased to $40(\mathrm{Gbit} / \mathrm{s}), 60(\mathrm{Gbit} / \mathrm{s}), 80(\mathrm{Gbit} / \mathrm{s})$, and $100(\mathrm{Gbit} / \mathrm{s})$, the maximum reliable transmission distance declines, such as 1400(km), $1300(\mathrm{~km}), 1250(\mathrm{~km}) 700(\mathrm{~km})$ respectively, as illustrated in Fig. 9b. Utilising the same example employed by (Paloi et al. 2017), for the $20(\mathrm{Gbit} / \mathrm{s})$, distance of $240(\mathrm{~km})$, and by observing all the data achieved from all simulations, we have designed graph Fig. 10 and achieved eye diagram graph Fig. 11a.

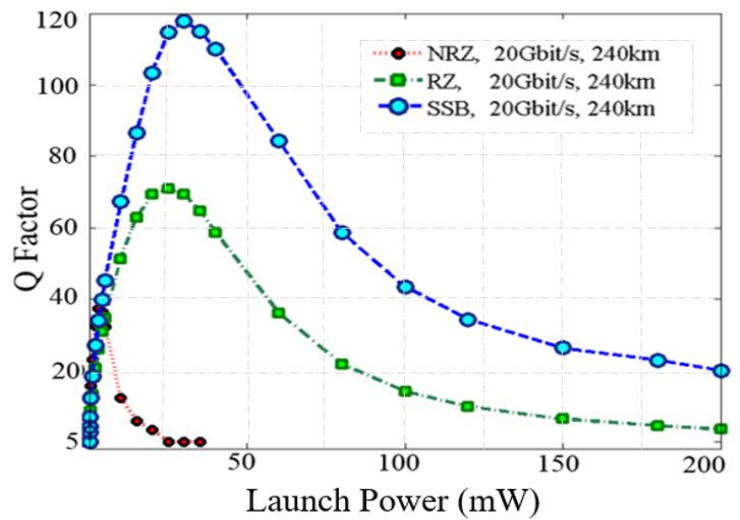

Fig. 10. Comparison of Q-Factor in function of launch power, between conventional NRZ, RZ and proposed SSB modulation scheme for 20 (Gbit/s) at the SSMF length of $240(\mathrm{~km})$.

The proposed SSB modulation format results with high peak power of $35(\mathrm{~mW})$, compared to 30 ( $\mathrm{mW}$ ) for the standard RZ and 5(mW) for the NRZ modulation format, as illustrated in Fig.10. When utilizing proposed SSB configuration for the length of $240(\mathrm{~km})$, at the launch power of $35(\mathrm{~mW})$, we have achieved Q-Factor of 120, compared to the conventional configuration used by Ref. (Miziya et al 2013; Gupa et al. 2012 and Paloi et al. 2017), where, for their peak power of $30(\mathrm{~mW})$ a maximum Q-Factor of 70 was achieved, as can be seen from Fig.10. Higher peak power means higher SNR, very high Q-Factor, and lower BER. This has been done in link with spur suppression, such as second and third order harmonics, and cancelling one of the sidebands. We can conclude that while requiring equal launch power, the proposed SSB structure results in a better performance, achieving nearly double Q-Factor, as is illustrated in the Fig.10 and achieved eye diagram Fig11a.
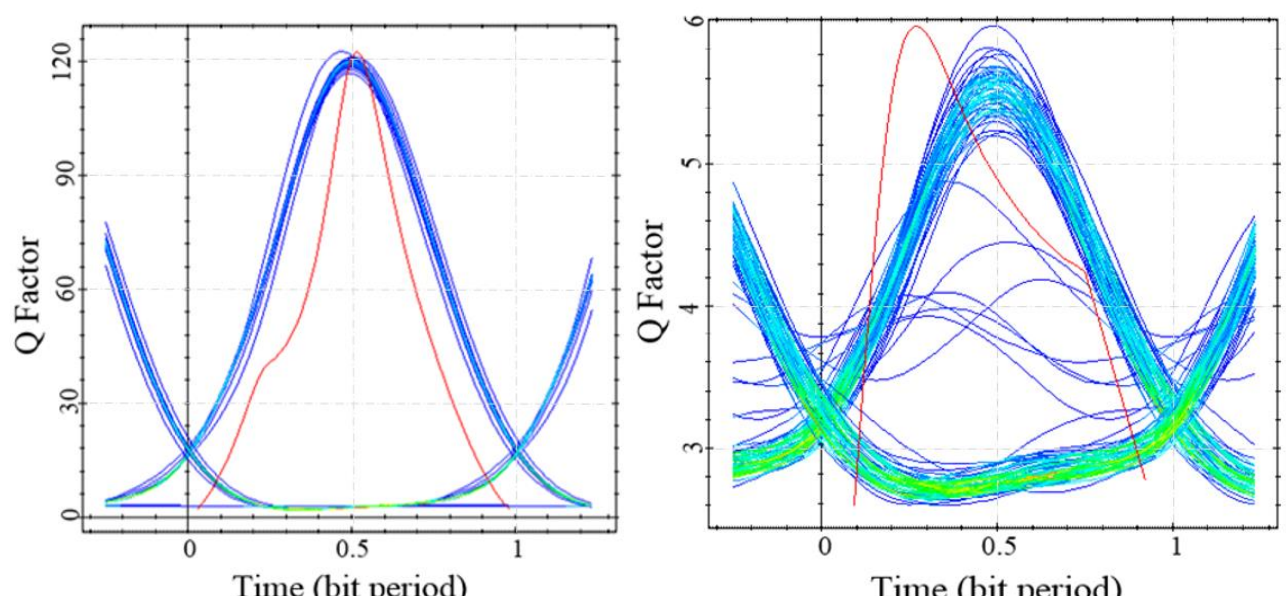

Fig. 11. Simulated eye diagram and achieved Q factor of proposed SSB modulation scheme, a) Symmetrical DCF at the length $240 \mathrm{~km}, 20 \mathrm{~Gb} / \mathrm{s}$. b) Without DCF and amplification for the length of $150 \mathrm{~km}, 10 \mathrm{~Gb} / \mathrm{s}$ data stream. 
Long haul optic transmission of lights over optical fibre represents excessive distance without or with minimum use of repeaters. The use of repeaters for the fibre optic cables can boost the signal at the interval along the transmission root. In a long-haul transmission, the main goal is to minimise the number of repeaters (Patel et al. 2014). The transmission performance of the OSSB modulated signal was investigated for various fibre lengths of $25(\mathrm{~km}), 50(\mathrm{~km}), 100(\mathrm{~km})$ up to $150(\mathrm{~km})$ of SMF link without any compensation, and no evident degradation was observed. With the proposed SSB configuration, we have utilised repeater only after $150(\mathrm{~km})$ for each span, where signal quality is kept stable during the transmission. Fig. $11 \mathrm{~b}$, shows simulated eye diagram and achieved Q-Factor (red curve) of 6 , corresponding BER of $10^{-9}$ for the length of $150(\mathrm{~km})$ without compensation and amplification.

The aim of the comparison in this paper was to measure the acceptance of SSB at various transmission distance. For long haul transmission of RoF signal over optical link it is necessary to compensate. We should choose the best and most cost-effective way to transmit the signal. We have utilised an SSB structure based on the RF demand and link distance, which can be used for the RoF system link. Both theoretical and simulating results show that the proposed SSB modulation format is solid, cost effective and performs better, graph Fig. 9b, as compared to previous standard modulation schemes (Paloi et al. 2017; Xu et al. 2015) graph Fig. 9a.

With proposed configuration, we have prevented nonlinear effects entering the link by suppressing harmonic distortions and using only a single sideband (Lim et al. 2007). Possibilities are halved because of the same bandwidth at half the power. With the SSB structure we can transmit signal up to $150 \mathrm{~km}$ without any compensation, Fig. 11b.

\section{Conclusion}

A theoretical analysis and simulation testing of the long-haul optical SSB modulation system has been demonstrated by using DP-DDMZM, biased at two different operating points. We have shown that, with the proposed configuration nonlinear effects at the source to the modulator have been prevented by using only a single sideband, where nonlinear possibilities are halved. We have demonstrated that the OSSB transmission can be used as a chromatic dispersion compensator in a long-haul transmission. In our proposed optical SSB modulation scheme, compensation and amplification have been used after $150(\mathrm{~km})$ for each span, and signal quality is being kept stable during the transmission, as compared to previous implementation where compensation and implementation is used at the distance of $100(\mathrm{~km})$ (Paloi et al. 2017; Xue et al. 2014). We have investigated existing optic fibre configuration systems, and we have managed to boost the optical signal by extending the length of the link from $2400(\mathrm{~km})$ to $3250(\mathrm{~km})$, with less system complexity at a lower cost. We have demonstrated that while requiring equal launch power, the proposed SSB structure shows more robustness against losses compared to a conventional modulation scheme, resulting in a better performance, where achieved Q-Factor is nearly doubled. Future work will address more robust transmission by suppressing more harmonics, including carrier suppression.

\section{References}

Hayashi, T., Tamura, Y., Hasegawa, T., et al.: Record-low Spatial Mode Dispersion and Ultra-Low Loss Coupled Multi-Core Fiber for Ultra-long-haul Transmission. Journal of Lightwave Technology, 35(3), 450-457 (2017).

Mizuno, T., Shibahara, K., Ye, F., Sasaki, Y, et al.: Long-Haul Dense Space Division Multiplexed Transmission Over Low-Crosstalk Heterogeneous 32-Core Transmission Line Using Partial Recirculating Loop System. Journal of Lightwave Technology, 35(3), 488-498 (2017).

Wei, C. C., Chen, K. Z., Chen, L. W., et al.: High-Capacity Carrier less Amplitude and Phase Modulation for WDM Long-Reach PON Featuring High Loss Budget. Journal of Lightwave Technology, 35(4), 1078-1082 (2017).

Haxha, S., Ademgil, H.: Novel Design of Photonic Crystal Fibres with Low Confinement Losses, Nearly Zero Ultra-Flatted Chromatic Dispersion, Negative Chromatic Dispersion and Improved Effective Mode Area. Journal of Optics Communications, 281(2), 278-286 (2008)

Hirano, M., Hagihara, S., Ohkubo, F., Koyano, Y., et al.: DCF module with low insertion loss, small residual dispersion, and low PMD, Conference on Optical Fibre Communication, San Diego, 1-3 Mar. 2009.

Niknamfar, M., Shadaram, M.: OSSB and ODSB configurations for RoF transmission over DWDM link. 15th International Conference on Transparent Optical Networks (ICTON), Cartagena, 1-4 Jun 2013

Phillips, M. R., Darcie, T. E.: Lightwave Analog Video Transmission, Optical Fiber Telecommunications IIIA, Kaminow, I. P., Koch, T. L. (eds.), pp. 523-559, Academic Press, San Diego (1997)

Chen, X., Yao, J.: A High Spectral Efficiency Coherent RoF System Based on OSSB Modulation with Low-Cost Free-Running Laser Sources for UDWDM-PONs. Journal of Lightwave Technology, 34 (11), 2789-2795 (2016). 
Miziya, K., Sudheer, S. K., Kuriakose, A. C.: Characterization of an optical communication system utilizing dispersion compensating fibre and nonlinear optical effects, Fourth International Conference on Computing, Communications and Networking Technologies, Tiruchengode, 1-6 Jul. 2013

Gupta, S., Shukla, N., Jaiswal, S.: Pre-, post, symmetric1 and 2 compensation techniques with RZ modulation. $1^{s t}$ International Conference on Recent Advances in Information Technology, Dhanbad, 251-255 May 2012.

Paloi, F., Mirza, T., Haxha, S.: Optimisation of dispersion compensating in a long-haul fibre for RF transmission of up to $100 \mathrm{Gbit} / \mathrm{s}$ by using RZ and NRZ formats. Optik - International Journal for Light and Electron Optics, 131, 640-654 (2017).

VPI Photonics, design automation.: http://vpiphotonics.com/index.php.

Optiwave Photonic Software.: https://optiwave.com/

Jiang, W., Tan, Q. Qin, W., et al.: A Linearization Analog Photonic Link with High Third-Order Intermodulation Distortion Suppression Based on Dual-Parallel Mach-Zehnder Modulator. IEEE Photonics Journal, 7(3) 18 (2015).

Devgan, P., Hastings, A., Urick, V. et al.: Cancellation of photodiode-induced second harmonic distortion using single side band modulation from a dual parallel Mach-Zehnder. Opt. Express, 20 (24) 27163-27173 (2012).

$\mathrm{Xu}, \mathrm{K} ., \mathrm{Ou}, \mathrm{Y}$.: Theoretical and numerical characterization of a $40 \mathrm{Gbit} / \mathrm{s}$ long-haul multi-channel transmission system with dispersion compensation. Digital Communications and Networks, 1 (3) 222-228 (2015).

Patel, A., Patel, R., Mehta, K.: Comparative analysis of single span high speed $40 \mathrm{Gbp} / \mathrm{s}$ long haul optical link using different modulation formats in the presence of Kerr nonlinearity. Students' Technology Symposium, IEEE, Kharagpur, 132-137, May 2014.

Breuer, D., Küppers, F., Mattheus, A., et al.: Symmetrical dispersion compensation for chromatic standard monomode-fibre communication systems with large amplifier spacing. Opt. Lett. 22 (13) 982-984 (1997).

Svarny, J.: Specialized compact external electro-optic modulating unit. 36th International Conference on Telecommunications and Signal Processing (TSP), Rome, 227-230 Sept. 2013

Li, Y., Zhang, Y., Huang, Y.: Any Bias Point Control Technique for Mach-Zehnder Modulator. IEEE Photonics Technology Letters, 25 (24) 2412-2415 (2013)

Freude, W., Schmogrow, R., Nebendahl, B., et al.: Quality metrics for optical signals: Eye diagram, Q-factor, OSNR, EVM and BER, 14 ${ }^{\text {th }}$ International Conference on Transparent Optical Networks, 1-4, Coventry 2012.

Hu, W.W., Inagaki, K., Tanaka, T., et al.: High SNR $50 \mathrm{GHz}$ radio-over-fibre uplink system by use of low biased Mach-Zehnder modulator technique. Electron. Lett., 42 (9), 550-552 (2006).

Yu, S., Gu, W., Yang, A., et al.: A Frequency Quadrupling Optical mm-Wave Generation for Hybrid FiberWireless Systems, IEEE Journal on Selected Areas in Communications, 31 (12), 797-803 (2013).

Cui, Y., Xu, K., Dai, J., et al.: Overcoming Chromatic-Dispersion-Induced Power Fading in ROF Links Employing Parallel Modulators. IEEE Photonics Technology Letters, 24 (14), 1173-1175 (2012).

Eappen, N. I., Sangeetha, A.: Analysis and performance comparison of inverse dispersion compensation technique for standard optical fibres, First International Conference on Computational Systems and Communications, 364-369, Dec. 2014

Xue, M., Pan S., Zhao, Y.: Optical Single-Sideband Modulation Based on a Dual-Drive MZM and a $120^{\circ}$ Hybrid Coupler. Journal of Lightwave Technology, 32 (19), 3317-3323 (2014)

Lin, C. T., Chen, J., Dai, S. P., Peng, P. C. and Chi, S.: Impact of Nonlinear Transfer Function and Imperfect Splitting Ratio of MZM on Optical Up-Conversion Employing Double Sideband with Carrier Suppression Modulation, Journal of Lightwave Technology, 26 (15), 2449-2459 (2008)

Ferreira J. A. A., Coutinho, O. L., Martins, C. S., Fegadolli, W. S., Ribeiro, J. A J. and Olivera, J. E. B.: Effect of Fibre Optic Chromatic dispersion on the performance of Analogue Optical Link with Dual-Drive MachZehnder Modulator, INTEL, ITA, 25 (28), 119-126 (2012)

Dattoli, G., Torre, A., Lorenzutta, S.: Theory of multivariable Bessel functions and elliptic modular functions $l e$ matematiche, 53(2), 387-399 (1998).

Kaur, J. and Sharma, N.: Effects of Amplified Spontaneous Emission (ASE) on NRZ, RZ and CSRZ modulation formats in single channel light-wave system, International Conference on Emerging Trends in Networks and Computer Communications, (ETNCC), Udaipur, 61-64, April 2011.

Thomas, V. A., El-Hajjar, M. and Hanzo, L.: Millimeter-Wave Radio Over Fiber Optical Upconversion Techniques Relying on Link Nonlinearity, IEEE Communications Surveys \& Tutorials, 18 (1), 29-53 (2016).

Rogers III, C. E., Carini, J. L., Pechkis, J. A. and Gould, P. L.: Characterization and compensation of the residual chirp in a Mach-Zehnder-type electro-optical intensity modulator, Optics Express, 18 (2), 1166-1176 (2010).

Shimotsu, S., Oikawa, S., Saitou, T., Mitsugi, N., Kubodera, K., Kawanishi, T. and Izutsu, M.: Single Side-Band Modulation Performance of a LiNbO3 Integrated Modulator Consisting of Four-Phase Modulator Waveguides, IEEE Photonics Technology Letters, 13(4), 364-366 (2001).

Lim, C., Nirmalathas, A., Lee, K-L., Novak, D. and Waterhouse, R.: Intermodulation distortion improvement for fiber-radio applications incorporating OSSB $+\mathrm{C}$ modulation in an optical integrated-access environment, $J$. Lightwave Technol. 25 (6) 1602-1612 (2007). 